\title{
THE SAFETY OF THE DENIS BROWNE ABDUCTION HARNESS IN CONGENITAL DISLOCATION OF THE HIP
}

\author{
CHRISTOPHER ELSWORTH, GEOFFREY WALKER
}

From Queen Mary's Hospital for Children, Carshalton

\begin{abstract}
The Denis Browne abduction harness was used in the management of 127 abnormal hips in 104 children at Queen Mary's Hospital for Children from 1966 to 1980, both as the initial treatment for unstable hips recognised soon after birth, and for children presenting later and whose hips first required reduction by gradual abduction in traction.

The incidence of significant avascular necrosis was $3.1 \%$, and occurred only in the primary treatment group, emphasising again the need for gentle care of infant hips. There have been no other significant complications and the appliance has functioned well.
\end{abstract}

In 1948 Denis Browne described an abduction harness for use in the management of children with congenital dislocation of the hip. A leather ring round each thigh was attached to a metal bar which was held against the sacrum by a belt. The rings maintained the hips in stable reduction after initial treatment by manipulation and a plaster spica. The child's hips were held in wide abduction, but could move relatively freely; walking was possible in the harness. The controlled mobility provided for the hip was considered to be an advantage over prolonged immobilisation in plaster.

A modification of this harness has been used at Queen Mary's Hospital for Children from 1966 until 1980. It has a sacral pad to prevent chafing, and shoulder straps with Velcro fasteners. The rings are covered with latex rubber and are sewn around the child's thighs by the nursing staff in order to prevent unauthorised removal at any stage of treatment. When necessary, change of harness was carried out by hospital nursing staff under medical supervision (Figs 1 and 2).

The Denis Browne harness is very useful in maintaining reduction, however this was originally achieved, but one of the major problems of the management is avascular necrosis of the femoral head. The main purpose of this study was to determine whether the use of the Denis Browne harness produces this serious complication. We also considered other less significant complications which might occur with this abduction apparatus.

C. Elsworth, FRCS, Senior Orthopaedic Registrar

Withington Hospital, West Didsbury, Manchester M20 8LR, England. G. Walker, FRCS, Orthopaedic Surgeon

Queen Mary`s Hospital for Children. Carshalton, Surrey SM5 4NR, England.

Requests for reprints should be sent to $\mathrm{Mr} \mathrm{C}$. Elsworth.

c 1986 British Editorial Society of Bone and Joint Surgery $0301-620 \times 86 \quad 2012 \$ 2.00$

\section{METHOD AND MATERIALS}

A study was made at Queen Mary's Hospital for Children of the case records and radiographs of all 104 children with congenital hip displacement who at any stage between 1966 and 1980 were treated with the Denis Browne abduction harness. The children either had an unstable hip at birth, or presented later with congenital dislocation, or had hip dysplasia recognised after the first few months of life. We also included one child with a diagnosis of "tight adductors" but normal radiographs. The management of these hips fell naturally into two groups: in one group a Denis Browne harness was used as primary treatment, in the other the harness was applied only after reduction had been achieved by gradual abduction on an overhead Eric Lloyd loop with or without cross-traction.

Primary treatment group. In all, 78 hips in 60 children. found to be unstable but reducible, usually detected within a few days of birth, were treated initially with an abduction harness (Fig. 3) which was worn for four to six months (average 5.3 months). Also treated and included in this group were three children with dysplastic acetabula in whom wide effortless abduction in flexion of the hips was possible after eight months of age.

Secondary treatment group. The Denis Browne harness was used for 44 children with 49 "abnormal" hips after gentle reduction by gradually increasing abduction in flexion. An overhead loop was used for up to four weeks, tight adductors were released by closed tenotomy, and cross-traction was occasionally necessary. All the hips were examined under anaesthesia at the time of application of a plaster spica; after six weeks this spica was replaced by the harness. The average age on application of the harness was greater in the secondary treatment group (Fig. 4) and in this group it was worn for 3 to 12 months (average 5.5 months).

After the harness had been removed hip movement 


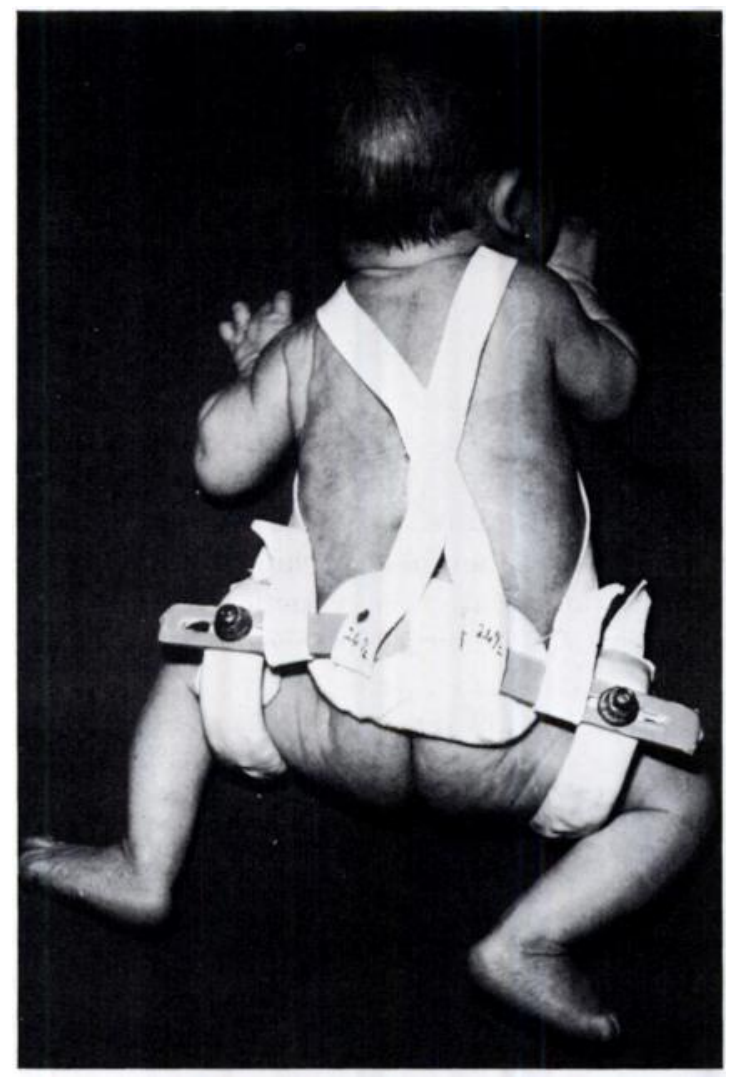

Fig. 1

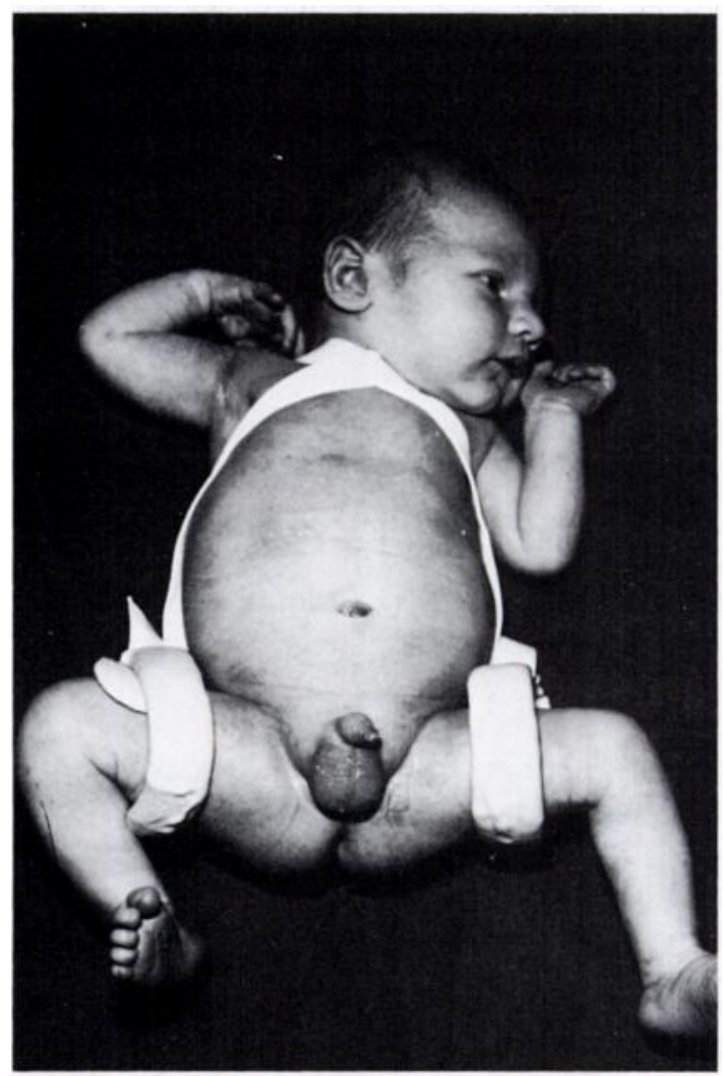

Fig. 2

The Denis Browne harness in use.

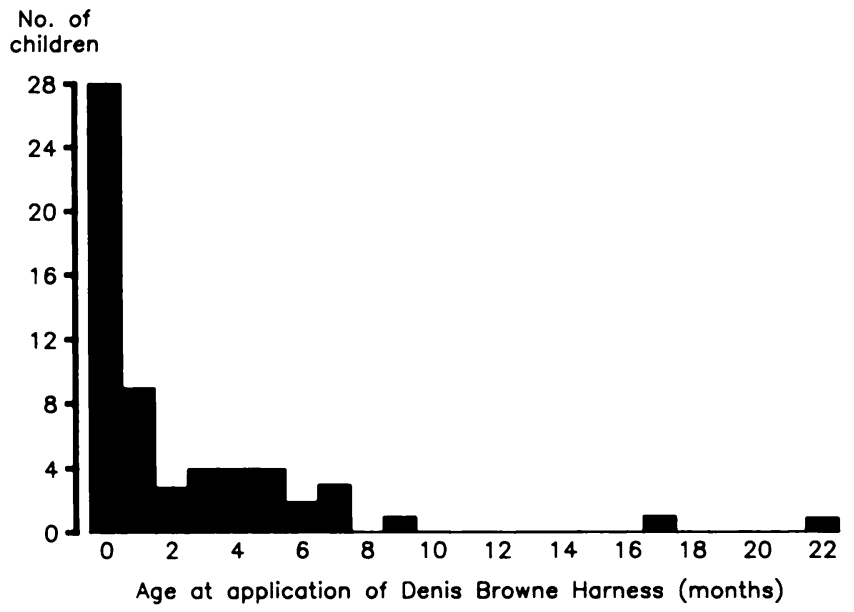

Fig. 3

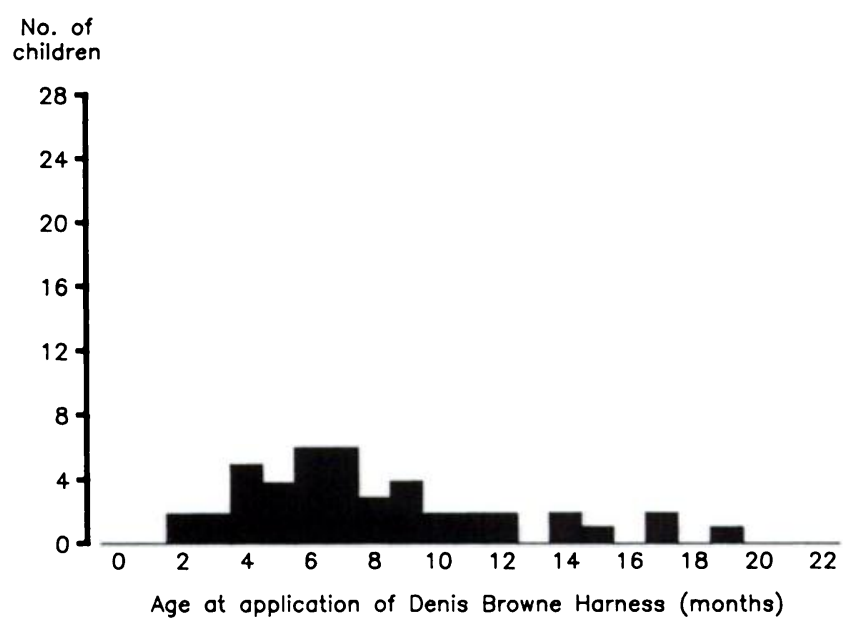

Fig. 4

Age in months at which the Denis Browne harness was first applied in the primary treatment group.

Age in months at which the Denis Browne harness was first applied in the secondary treatment group. 
and general mobilisation was unrestricted. All children were reviewed regularly until they were skeletally mature and radiographs were taken when they were indicated. The radiological features of avascular necrosis of the femoral head were described by Salter, Kostuik and Dallas in 1969. We assessed our radiographs using the grouping described by Kalamchi and MacEwen (1980). These groups are:

Group I. Changes in the capital femoral epiphysis only. Group II. Changes in the capital femoral epiphysis and in the lateral metaphysis.

Group III. Changes in the capital femoral epiphysis and the central metaphysis.

Group IV. Severe damage to the capital femoral epiphysis and to the metaphysis.

\section{RESULTS}

Of the 78 unstable hips in the 60 children in the primary treatment group, radiographic changes suggested avascular necrosis in seven hips, with none in a "stable" or "normal" joint. Three of the seven had Group I changes, two were in Group II and two in Group III. Of the 49 abnormal hips in the secondary treatment group of 44 children, there were radiographic changes of Group I severity in five, with none in a "normal" hip.

All eight hips which showed Group I avascular changes have, as expected, either returned or are returning to radiographic normality. Of the two primarily treated unstable hips with Group II avascular necrosis, one will certainly remain abnormal at skeletal maturity. The other has undoubted lateral metaphyseal involvement but since she was only eight years old at last review, the final outcome remains unclear. One of the hips with Group III changes will certainly be deformed at skeletal maturity; the other had changes in the capital epiphysis and central metaphysis when lost to follow-up at two years of age, but is likely to have permanent epiphyseal damage.

In total, of 127 hips, 12 had radiographic evidence of avascular necrosis. At the time of review only four of these were causing concern, two of which will have undoubted deformity of the mature femoral head. It is of considerable interest that there was no avascular necrosis in any of the "normal" hips, and even more important that no hips showed the severe changes of Group IV.

Other problems. While it is true that doctors and not splints treat congenital dislocation of the hip, it is possible to leave one or both unstable hips in the dislocated position when applying a Denis Browne harness. This did occur with six of the hips in our primary treatment group; five were recognised within two weeks and corrected. In the sixth child, dislocation remained unrecognised for four months despite great concern and care, and an open operation was required to correct the position. In the secondary treatment group no hips were splinted in the dislocated position.

Six children developed sores from the thigh rings; one of these, though relatively small, needed treatment by excision and suture. Other skin problems were managed satisfactorily by simple conservative means.

In one case only the harness was removed by the parents; this child was subsequently lost to follow-up.

\section{DISCUSSION}

At Queen Mary`s Hospital for Children, 14 years`experience with the Denis Browne abduction harness has shown it to be a useful and relatively safe means of maintaining stability after the reduction of congenitally dislocated hips. Of 127 hips, 12 showed radiographic evidence of avascular necrosis at some stage, but at late review only four caused concern, a $3.1 \%$ incidence of significant necrosis, all in the primary treatment group. It is difficult to compare this with other series, especially as we have not been able to find any record of another series of cases treated by the Denis Browne harness. The rate of avascular necrosis compares favourably, however, with that in other published series of the late results in assorted groups of children treated by various means (Salter $e t$ al. 1969; Gage and Winter 1972). Our findings, however, emphasise yet again that unstable neonatal hips must be treated with great gentleness.

Since 1980 the harness used at Queen Mary's Hospital for Children has been further modified to resemble the model used at the Rowley Bristow Hospital, Pyrford, which has a curved bar to allow better hip radiography and is known locally as the "Pyrford harness".

Our thanks are due to the Medical Illustration Departments at Queen Mary`s Hospital for Children. Carshalton and Manchester Royal Infirmary, and to Miss Susan Wadd for secretarial assistance.

\section{REFERENCES}

Browne D. The treatment of congenital dislocation of the hip. Proc $R$ Soc Med 1948:41:388-90.

Gage JR, Winter RB. Avascular necrosis of the capital femoral epiphysis as a complication of closed reduction of congenital dislocation of the hip: a critical review of twenty years' experience at Gillette Children's Hospital. J Bone Joint Surg [Am] 1972:54-A:373-88.

Kalamchi A, MacEwen GD. Avascular necrosis following treatment of congenital dislocation of the hip. J Bone Joint Surg $[\mathrm{Am}]$ 1980; 62-A : 876-8

Salter RB, Kostuik J, Dallas S. Avascular necrosis of the femoral head as a complication of treatment for congenital dislocation of the hip in young children: a clinical and experimental investigation. $C a n J$ Surg 1969:12:44 61. 